\title{
A GENERALIZED DUALITY TRANSFORMATION OF THE ANISOTROPIC $X Y$ CHAIN IN A MAGNETIC FIELD
}

\author{
Haye Hinrichsen \\ Universität Bonn, Physikalisches Institut \\ Nußallee 12, D-53115 Bonn, Germany
}

\begin{abstract}
We consider the anisotropic $X Y$ chain in a magnetic field with special boundary conditions described by a two-parameter Hamiltonian. It is shown that the exchange of the parameters corresponds to a similarity transformation, which reduces in a special limit to the Ising duality transformation.
\end{abstract}

BONN HE-93-40

cond-mat/9310055

October 1993 
In this paper we consider the anisotropic $X Y$ chain in a magnetic field which is defined by the Hamiltonian

$$
H^{X Y}(\eta, q)=-\frac{1}{2} \sum_{j=1}^{L-1}\left(\eta \sigma_{j}^{x} \sigma_{j+1}^{x}+\eta^{-1} \sigma_{j}^{y} \sigma_{j+1}^{y}+q \sigma_{j}^{z}+q^{-1} \sigma_{j+1}^{z}\right),
$$

where $q$ and $\eta$ are complex parameters and $\sigma_{j}^{x, y, z}$ are Pauli matrices acting on site $j$. Up to boundary terms, which will play a crucial role here, $H$ can be rewritten as

$$
H^{X Y}(\eta, h)=-\frac{1}{2} \sum_{j=1}^{L}\left(\eta \sigma_{j}^{x} \sigma_{j+1}^{x}+\eta^{-1} \sigma_{j}^{y} \sigma_{j+1}^{y}\right)-h \sum_{j=1}^{L} \sigma_{j}^{z}
$$

where $h=\frac{q+q^{-1}}{2}$ is the magnetic field. This Hamiltonian has a long history [1, 2] and provides a good model for Helium adsorbed on metallic surfaces ( $\eta$ real and $q$ on the unit circle). It also gives the master equation of the kinetic Ising model [3] ( $q=1$ and $\eta$ real) and plays a role in one-dimensional reaction-diffusion processes [4]. For the special boundary conditions defined in Eq. (11) the chain has been shown to be invariant under a two-parameter deformation of the $s u(1 \mid 1)$ superalgebra [5], and some of their correlation functions in the massless regime have been computed in Ref. [6].

The aim of this paper is to show that for these boundary conditions the exchange of the parameters $q$ and $\eta$ in the Hamiltonian (四) corresponds to a similarity transformation

$$
H^{X Y}(\eta, q) \doteq H^{X Y}(q, \eta)
$$

which reduces in a special limit to the Ising duality transformation (here ' $\doteq$ ' denotes equality up to a similarity transformation). The Ising limit of the $X Y$ chain is given by

$$
H^{I S}(a, b)=\lim _{\xi \rightarrow \infty} \frac{1}{\xi} H^{X Y}(a \xi, b \xi)
$$

where

$$
H^{I S}(a, b)=-\frac{1}{2} \sum_{j=1}^{L-1}\left(a \sigma_{j}^{x} \sigma_{j+1}^{x}+b \sigma_{j}^{z}\right)
$$

is the Ising Hamiltonian with mixed boundary conditions [7]. One of the most remarkable properties of the Ising model is its self-duality [8]. For the boundary conditions defined in Eq. (5) the Ising duality transformation

$$
\sigma_{j}^{x} \rightarrow \tilde{\sigma}_{j}^{x}=\prod_{i=1}^{j} \sigma_{i}^{z}, \quad \sigma_{j}^{z} \rightarrow \tilde{\sigma}_{j}^{z}=\sigma_{j}^{x} \sigma_{j+1}^{x}
$$

takes place as

$$
H^{I S}(a, b) \doteq H^{I S}(b, a)+a\left(\sigma_{L}^{z}-\sigma_{1}^{z}\right) .
$$

Using Eq. (州) we can rewrite Eq. (7) by

$$
\lim _{\xi \rightarrow \infty} \frac{1}{\xi} H^{X Y}(a \xi, b \xi) \doteq \lim _{\xi \rightarrow \infty} \frac{1}{\xi} H^{X Y}\left(b \xi, \frac{1}{a \xi}\right) .
$$

Notice that we absorbed the surface terms in Eq. (7) by inserting the argument $\frac{1}{a \xi}$ instead of $a \xi$ on the r.h.s. of Eq. (8). In order to symmetrize this expression, we perform a rotation

$$
\sigma_{j}^{x} \rightarrow \sigma_{j}^{y}, \quad \sigma_{j}^{y} \rightarrow-\sigma_{j}^{x}, \quad \sigma_{j}^{z} \rightarrow \sigma_{j}^{z}, \quad(j=1, \ldots, L)
$$


on the 1.h.s. of Eq. (8):

$$
H^{X Y}(a \xi, b \xi) \doteq H^{X Y}\left(\frac{1}{a \xi}, b \xi\right)
$$

and we obtain

$$
\lim _{\xi \rightarrow \infty} \frac{1}{\xi} H^{X Y}\left(\frac{1}{a \xi}, b \xi\right) \doteq \lim _{\xi \rightarrow \infty} \frac{1}{\xi} H^{X Y}\left(b \xi, \frac{1}{a \xi}\right) .
$$

This means that Eq. (3) holds for $\eta=\frac{1}{a \xi}$ and $q=b \xi$ in the limit $\xi \rightarrow \infty$.

The claim of this paper is to prove Eq. (3)), i.e. we derive a similarity transformation

$$
H^{X Y}(\eta, q)=U H^{X Y}(q, \eta) U^{-1}
$$

for arbitrary parameters $\eta$ and $q$. Let us first summarize our results. For that purpose let us introduce fermionic operators by a Jordan-Wigner transformation

$$
\tau_{j}^{x, y}=\left(\prod_{i=1}^{j-1} \sigma_{i}^{z}\right) \sigma_{j}^{x, y}
$$

which allows the Hamiltonian (11) to be written as

$$
H(\eta, q)=\frac{i}{2} \sum_{j=1}^{L-1}\left(\eta \tau_{j}^{-} \tau_{j+1}^{+}-\eta^{-1} \tau_{j}^{+} \tau_{j+1}^{-}+q \tau_{j}^{+} \tau_{j}^{-}+q^{-1} \tau_{j+1}^{+} \tau_{j+1}^{-}\right) .
$$

Denoting

$$
\alpha=\frac{q}{\eta}, \quad \omega=\left(\frac{\alpha^{1 / 2}-\alpha^{-1 / 2}}{\alpha^{1 / 2}+\alpha^{-1 / 2}}\right)
$$

the explicit expression for $U(\alpha)$ is given by the polynomial

$$
U(\alpha)=\frac{1}{\sqrt{N}} \sum_{k=0}^{[L / 2]} \omega^{k} G_{2 k}
$$

where the generators $G_{2 k}$ are defined by

$$
G_{n}=\sum_{1 \leq j_{1}<j_{2}<\ldots<j_{n} \leq L} \tau_{j_{1}}^{x} \tau_{j_{2}}^{x} \ldots \tau_{j_{n}}^{x} .
$$

By convention we take $G_{0} \equiv \mathbf{1}$, and $[L / 2]$ denotes the truncation of $L / 2$ to an integer number. $N$ is a normalization constant which is given by

$$
N=\sum_{k=0}^{[L / 2]}\left(\begin{array}{c}
L \\
2 k
\end{array}\right) \omega^{2 k}=2^{L-1} \frac{1+\alpha^{L}}{(1+\alpha)^{L}} .
$$

Notice that the transformation depends only on the ratio $\alpha=q / \eta$. Obviously the normalization $N$ vanishes for $\alpha^{L}=-1$ so that the transformation (12) diverges, and therefore we will exclude this case in the following. For $\alpha=1$ the transformation $U(\alpha)$ reduces to the identity, and this is what we expect since for $\eta=q$ the exchange of $\eta$ and $q$ does not effect a change in the Hamiltonian (四).

In order to express the transformation in a formal way, let us introduce the 'time-ordered product'

$$
T \tau_{i}^{x} \tau_{j}^{x}=\left\{\begin{array}{cc}
\tau_{i}^{x} \tau_{j}^{x} & i<j \\
-\tau_{j}^{x} \tau_{i}^{x} & i>j \\
0 & i=j
\end{array}\right\}
$$


which arranges the operators $\tau_{j}^{x}$ in increasing order with respect to their fermionic commutation relations. Observing that

$$
G_{2 k}=\frac{1}{k !} T G_{2}^{k}
$$

where

$$
G_{2}=\sum_{1 \leq j_{1}<j_{2} \leq L} \tau_{j_{1}}^{x} \tau_{j_{2}}^{x}
$$

we can rewrite Eq. (16) formally as a time-ordered exponential of $G_{2}$ :

$$
U(\alpha)=\frac{1}{\sqrt{N}} T \exp \left(\omega G_{2}\right)
$$

This expression suggests that the inverse of $U(\alpha)$ is obtained by taking $\omega \rightarrow-\omega$, i.e. $\alpha \rightarrow \alpha^{-1}$. In fact, one can show that

$$
U^{-1}(\alpha)=U\left(\alpha^{-1}\right)
$$

On the other hand we observe that $G_{2}^{T}=-G_{2}$ and thus the transformation (12) is an orthogonal one:

$$
U^{T}(\alpha)=U^{-1}(\alpha)
$$

It is interesting to know how the Pauli matrices change under the transformation

$$
\tilde{\sigma}_{j}^{x, y, z}=U(\alpha) \sigma_{j}^{x, y, z} U^{-1}(\alpha) .
$$

As we are going to show below, one obtains three important identities:

$$
\begin{aligned}
\eta \tilde{\sigma}_{j}^{x} \tilde{\sigma}_{j+1}^{x}+q \tilde{\sigma}_{j}^{z} & =q \sigma_{j}^{x} \sigma_{j+1}^{x}+\eta \sigma_{j}^{z} \\
q \tilde{\sigma}_{j}^{y} \tilde{\sigma}_{j+1}^{y}+\eta \tilde{\sigma}_{j+1}^{z} & =\eta \sigma_{j}^{y} \sigma_{j+1}^{y}+q \sigma_{j+1}^{z} \\
\tilde{\sigma}_{j}^{x} \tilde{\sigma}_{j+1}^{y} & =\sigma_{j}^{x} \sigma_{j+1}^{y} .
\end{aligned}
$$

Because of these identities we have

$$
\begin{aligned}
\tilde{H}^{X Y}(q, \eta) & =-\frac{1}{2} \sum_{j=1}^{L-1}\left[q \tilde{\sigma}_{j}^{x} \tilde{\sigma}_{j+1}^{x}+q^{-1} \tilde{\sigma}_{j}^{y} \tilde{\sigma}_{j+1}^{y}+\eta \tilde{\sigma}_{j}^{z}+\eta^{-1} \tilde{\sigma}_{j+1}^{z}\right] \\
& =-\frac{1}{2} \sum_{j=1}^{L-1}\left[\eta \sigma_{j}^{x} \sigma_{j+1}^{x}+\eta^{-1} \sigma_{j}^{y} \sigma_{j+1}^{y}+q \sigma_{j}^{z}+q^{-1} \sigma_{j+1}^{z}\right]=H^{X Y}(\eta, q),
\end{aligned}
$$

and our claim in Eq. (3) is proved. The identities (26)-(28) contain even more information. Since they hold independently for every $1 \leq j<L$, it is obvious that even the spectrum of the Hamiltonian

$$
\bar{H}=-\frac{1}{2} \sum_{j=1}^{L-1}\left[a_{j}\left(\eta \sigma_{j}^{x} \sigma_{j+1}^{x}+q \sigma_{j}^{z}\right)+b_{j}\left(\eta^{-1} \sigma_{j}^{y} \sigma_{j+1}^{y}+q^{-1} \sigma_{j+1}^{z}\right)+c_{j} \sigma_{j}^{x} \sigma_{j+1}^{y}\right]
$$

is invariant under the exchange $q \leftrightarrow \eta$ for arbitrary coefficients $a_{j}, b_{j}$ and $c_{j}$.

We are now going to derive the identities (26)-(28). For that purpose we first consider the transformation properties of the fermionic operators $\tilde{\tau}_{j}^{x, y}=U \tau_{j}^{x, y} U^{-1}$. It turns out that

$$
\tilde{\tau}_{i}^{x}=\sum_{i=1}^{L} u_{i, j} \tau_{j}^{x}, \quad \tilde{\tau}_{j}^{y}=\tau_{j}^{y}
$$

where

$$
u_{i, j}=\left\{\begin{array}{ll}
\varrho & \text { if } i=j \\
(\varrho-\alpha) \alpha^{i-j} & \text { if } i<j \\
\left(\varrho-\alpha^{-1}\right) \alpha^{i-j} & \text { if } i>j
\end{array}\right\}
$$


and

$$
\varrho=\frac{\alpha^{\frac{L}{2}-1}+\alpha^{-\frac{L}{2}+1}}{\alpha^{\frac{L}{2}}+\alpha^{-\frac{L}{2}}} .
$$

Notice that the transformation (31) is an orthogonal one as well $\left(\sum_{k=1}^{L} u_{i, k} u_{j, k}=\delta_{i, j}\right)$ and reduces for $\alpha=1$ to the identical transformation $u_{i, j}=\delta_{i, j}$. Furthermore the coefficients $u_{i, j}$ depend only on the difference of their indices $i-j$. Obviously $\tau_{j}^{y}$ is invariant under the similarity transformation, and this immediately proves Eq. (28). By adding the JordanWigner transformation (13) we obtain the following transformation rules for other the terms occuring in the Hamiltonian:

$$
\begin{aligned}
& \tilde{\sigma}_{j}^{x} \tilde{\sigma}_{j+1}^{x}=\left(\varrho-\alpha^{-1}\right) \sum_{k=1}^{j-1} \alpha^{j-k+1} \sigma_{k}^{y} S_{k+1, j-1} \sigma_{j}^{y} \\
& +(\varrho-\alpha) \sum_{k=j+2}^{L} \alpha^{j-k+1} \sigma_{j}^{x} S_{j+1, k-1} \sigma_{k}^{x} \\
& +(1-\varrho \alpha) \sigma_{j}^{z}+\varrho \sigma_{j}^{x} \sigma_{j+1}^{x} \\
& \tilde{\sigma}_{j}^{y} \tilde{\sigma}_{j+1}^{y}=\left(\varrho-\alpha^{-1}\right) \sum_{k=1}^{j-1} \alpha^{j-k} \sigma_{k}^{y} S_{k+1, j} \sigma_{j+1}^{y} \\
& +(\varrho-\alpha) \sum_{k=j+2}^{L} \alpha^{j-k} \sigma_{j+1}^{x} S_{j, k-1} \sigma_{k}^{x} \\
& +\left(1-\varrho \alpha^{-1}\right) \sigma_{j+1}^{z}+\varrho \sigma_{j}^{y} \sigma_{j+1}^{y} \\
& \tilde{\sigma}_{j}^{z}=\left(\alpha^{-1}-\varrho\right) \sum_{k=1}^{j-1} \alpha^{j-k} \sigma_{k}^{y} S_{k+1, j-1} \sigma_{j}^{y} \\
& +(\alpha-\varrho) \sum_{k=j+1}^{L} \alpha^{j-k} \sigma_{j}^{x} S_{j+1, k-1} \sigma_{k}^{x}+\varrho \sigma_{j}^{z}, \\
& \tilde{\sigma}_{j}^{y} \tilde{\sigma}_{j+1}^{x}=\left(\varrho-\alpha^{-1}\right) \sum_{k=1}^{j-1} \alpha^{j-k}\left(\alpha \sigma_{k}^{y} S_{k+1, j} \sigma_{j+1}^{x}-\sigma_{k}^{y} S_{k+1, j-1} \sigma_{j}^{x}\right) \\
& +(\varrho-\alpha) \sum_{k=j+2}^{L} \alpha^{j-k}\left(\sigma_{j}^{y} S_{j+1, k-1} \sigma_{k}^{x}-\alpha \sigma_{j+1}^{y} S_{j+2, k-1} \sigma_{k}^{x}\right) \\
& +\left(\varrho\left(\alpha+\alpha^{-1}\right)-1\right) \sigma_{j}^{y} \sigma_{j+1}^{x},
\end{aligned}
$$

Here $S_{i, k}$ denotes the Jordan-Wigner string between the sites $i$ and $k$

$$
S_{i, k}=\prod_{j=i}^{k} \sigma_{j}^{z}, \quad S_{i+1, i} \equiv 1
$$

which means that the $q \leftrightarrow \eta$ transformation converts local observables to linear combinations of strings measuring the charge between certain positions. Notice that Eqs. (34)-(37) simplify in the thermodynamical limit $L \rightarrow \infty$, where $\varrho \rightarrow \alpha$ if $|\alpha|<1$ and $\varrho \rightarrow \alpha^{-1}$ if $|\alpha|>1$, respectively. 
We have discovered the identity (12) by first noticing that the spectra of $H^{X Y}(\eta, q)$ and $H^{X Y}(q, \eta)$ are identical and we have computed the relations (34)-(37) by hand. Then using these results we conjectured the general structure of the transformation (16).

Let us finally check the Ising limit described above (c.f. Eq. (11)). For $q \rightarrow \infty$ and $\eta \rightarrow 0$ Eqs. (26) and (27) reduce to

$$
\tilde{\sigma}_{i}^{z}=\sigma_{i}^{x} \sigma_{i+1}^{x}, \quad \tilde{\sigma}_{i}^{y} \tilde{\sigma}_{i+1}^{y}=\sigma_{i+1}^{z}
$$

Now if we rotate $\tilde{\sigma}^{x}$ and $\tilde{\sigma}^{y}$ like in Eq. (9), we end up with

$$
\tilde{\sigma}_{i}^{z}=\sigma_{i}^{x} \sigma_{i+1}^{x}, \quad \tilde{\sigma}_{i}^{x} \tilde{\sigma}_{i+1}^{x}=\sigma_{i+1}^{z},
$$

and this is just the Ising duality transformation given in Eq. (6).

The $q \leftrightarrow \eta$ symmetry (29) may be interpreted physically as follows: The parameter $q$ fixes (beyond the magnetic field) the boundary conditions of the system while the parameter $\eta$ describes the bulk anisotropy. So the exchange of $q$ and $\eta$ may be understood as a transformation which exchanges the bulk and boundary properties of the chain. An investigation of correlation functions confirms this interpretation.

\section{Acknowledgements}

I would like to thank V. Rittenberg for valuable discussions and S. R. Dahmen for a careful reading of the manuscript.

\section{References}

[1] den Nijs N 1988 Phase Transitions and Critical Phenomena ed Domb C and Lebowitz J L, vol. 12 (Academic Press New York) 219

[2] Barouch E, McCoy B M and Dresden M 1970 Phys. Rev. A2 1075 Barouch E and McCoy B M 1971 Phys. Rev. A3 786

[3] Siggia E D 1977 Phys. Rev. B16 2319

[4] Alcaraz F C, Droz M, Henkel M and Rittenberg V 1992 Reaction-Diffusion Processes, Critical Dynamics and Quantum Chains Geneva preprint UGVA-DPT 1992/12-799

[5] Saleur H 1989 Trieste Conference on Recent Developements in Conformal Field Theories ed Randjikar-Daemi, E. Sezgin and J. B. Zuber (World Scientific) 160 Hinrichsen H and Rittenberg V 1992 Phys. Lett. B 275350

[6] Hinrichsen H and Rittenberg V 1993 Phys. Lett. B 304115

[7] Cardy J L 1986 Nucl. Phys. B 275200

[8] Kogut J B 1979 Rev. Mod. Phys 51659 